\title{
Modifications of Bordetella bronchiseptica core lipopolysaccharide influence immune response without affecting protective activity
}

\author{
Federico Sisti $^{\mathrm{a}, *}$, Julieta Fernández ${ }^{\mathrm{a}}$, Andrés Cordero ${ }^{\mathrm{a}}$, Adriana Casabuono ${ }^{\mathrm{b}}$, Alicia Couto ${ }^{\mathrm{b}}$, Daniela Hozbor ${ }^{\mathrm{a}}$

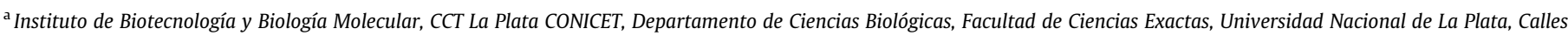 \\ 47 y 115, 1900 La Plata, Argentina \\ ${ }^{\mathrm{b}}$ CIHIDECAR, Departamento de Química Orgánica, Facultad de Cs Exactas y Naturales, Universidad de Buenos Aires, 1428 Bs. As., Argentina
}

\section{A R T I C L E I N F O}

\section{Article history:}

Received 4 October 2016

Revised 18 December 2016

Accepted 19 December 2016

Available online 21 December 2016

\section{Keywords:}

Lipopolysaccharide

Bordetella

Glycosyltransferase

\begin{abstract}
A B S T R A C T
Bordetella bronchiseptica produces respiratory disease primarily in mammals including humans. Although a considerably amount of research has been generated regarding lipopolysaccharide (LPS) role during infection and stimulating innate and adaptive immune response, mechanisms involved in LPS synthesis are still unknown. In this context we searched in B. bronchiseptica genome for putative glycosyltransferases. We found possible genes codifying for enzymes involved in sugar substitution of the LPS structure. We decided to analyse BB3394 to BB3400 genes, closed to a previously described LPS biosynthetic locus in B. pertussis. Particularly, conservation of BB3394 in sequenced $B$. bronchiseptica genomes suggests the importance of this gene for bacteria normal physiology. Deletion of BB3394 abolished resistance to naive serum as described for other LPS mutants. When purified LPS was analyzed, differences in the LPS core structure were found. Particularly, a GalNA branched sugar substitution in the core was absent in the LPS obtained from BB3394 deletion mutant. Absence of GalNA in core LPS alters immune response in vivo but is able to induce protective response against $B$. bronchiseptica infection.
\end{abstract}

(c) 2016 Published by Elsevier Ltd.
Bacterial surface polysaccharides are known to contribute to bacterium-host interaction, including symbiotic and pathogenic relationships. ${ }^{1}$ Among these polysaccharides, the lipopolysaccharide (LPS) is the major component of the outer membrane of gram-negative bacteria and consists of highly variable as well as conserved segments. ${ }^{2}$ In extremely small amounts LPS possess potent bioactivities initiating the morbidity and mortality associated with Gram-negative sepsis as well as the modulation of a myriad of host innate inflammatory responses. Interestingly, specific synthetic alterations within conserved segments of LPS were shown to be recognized not only by host cells but were also capable of blocking or down-regulating the response to other LPS

Abbreviations: Kdo, 3-deoxy-D-manno-octulosonic acid; Hep, Heptose, L-glycero-Dmanno-heptose; GlcA, glucuronic acid; GlcN, glucosamine; GlcNAc, N-acetylglucosamine; FucN, Fuc2NAc4N, 2-acetamido 4-amino 2,4,6-trideoxy-galactose; Man2,3NAcA, 2, 3-diacetamido-2,3-dideoxymannuronic acid; GlcNAc, N-acetylglucosamine; Man2, 3NAcAN, 2,3-diacetamido-2,3-dideoxymannuronamide; GalNAc3NAcA, 2,3-diacetamido-2,3-dideoxygalacturonic acid; GlcNAc3NAcAN, 2,3-diacetamido-2,3-dideoxyglucuronamide; Lac type, 2-Nacetyl, 3-Nformyl, 4N-(2 metoxypropionyl)-2,3,4-triamino 2,3,4-trideoxy- $\alpha$-galacturonamide.

* Corresponding author at: Instituto de Biotecnología y Biología Molecular, CCT La Plata CONICET, Departamento de Ciencias Biológicas, Facultad de Ciencias Exactas, Universidad Nacional de La Plata, Calles 49 y 115, 1900 La Plata, Argentina.

E-mail address: federico@biol.unlp.edu.ar (F. Sisti). forms normally associated with robust innate cell activation. ${ }^{3}$ In fact, structural heterogeneity of LPS of certain pathogenic bacteria like Helicobacter pylori, was shown to result in differential and altered innate host cell responses. ${ }^{4-7}$

Bordetella bronchiseptica can infect a variety of mammals including humans. Other authors and ourselves previously showed that $B$. bronchiseptica expresses a LPS that plays an essential role in host interaction, being critical for the early clearance of the bacteria. $^{8}$ Like in other pathogens, $B$. bronchiseptica isolates from human patients presented LPS modifications. ${ }^{9}$ Purified $B$. bronchiseptica LPS presents after electrophoresis in polyacrylamide gels a pattern of three bands. Faster migrating band is called $\mathrm{B}$ and comprises a lipid A domain and a branched-chain core oligosaccharide. Band $B$ is further modified by the addition of a complex trisaccharide called band A (band B plus trisaccharide). In addition, a pentasaccharide links the $\mathrm{O}$ antigen consisting in a homopolymer of 2 , 3-dideoxy-2,3-di-N-acetylgalactosaminuronic acid to the coretrisaccharide region. ${ }^{10}$ The complete structure, (band B plus band A plus $\mathrm{O}$ antigen) is presented as the slower migrating band. Mutation of genes involved in core synthesis usually abolish further LPS synthesis. ${ }^{11,12}$ LPS structure in these mutants is called deep rough because of mutant colony morphology and is visualized in polyacrylamide gels as a single fast migrating band. 
The Bordetella LPS core presents one KDO molecule and two heptoses linked to lipid A. This composition is quite different from other LPSs where up to three heptoses are present. Only three genes, waaA, waaC and waaF are known to participate in the deep core synthesis. ${ }^{11,12}$ Guerten and co-workers described in B. pertussis four glycosyltransferases that participate in sugar core substitutions. However, no effects in Bordetella pathogenesis has been described for those mutants. ${ }^{13}$ We have previously reported that a deep rough LPS mutant is unable to establish an infection in wild type mice. ${ }^{12}$ Interestingly, we suggested that early elimination may be the strong response to a deep rough LPS compared to smooth LPS. Particularly an enhanced IL-12 and TNF-alpha response was observed in mice treated with a deep rough LPS suggesting that distal portions of LPS are involved in host immune response regulation. ${ }^{12}$

We hypothesized that enzymes that catalyse the addition of hexoses to the growing core of $B$. bronchiseptica LPS would be similar to known glycosyltransferases from other bacteria. Therefore, we found genes dispersed around all the B. bronchiseptica RB50 genome as shown in Supplemental Table S1. Pan-genome analysis indicates that genes associated with diverse phenotypes, antibiotic resistance or that confer selective advantages are often found within the accessory genome rather than the core genome. ${ }^{14}$ Hester and co-workers suggested that horizontally acquired divergent $\mathrm{O}$-antigen contributes to escape from cross-immunity in the classical Bordetellae. ${ }^{15}$ Dispersal position of these genes may suggest a possible source of LPS structure diversity between different Bordetella circulating strains. However, when other available genomes were analyzed, those genes were in the same relative position suggesting that glycosyltransferase genes are part of the core rather than accessory genome. Some of those genes are arranged in groups of putative glycosyltransferases. One of those groups, involving BB3396 to BB3400 are homologous to BP2328 to BP2331 previously described by Geurtsen and co-workers as glycosyltransferases involved in B. pertussis Tohama I LPS core synthesis. ${ }^{13}$ Moreover, a similar group of genes in Burkholderia cenocepacie were previously described. ${ }^{16}$ Interestingly, one of $B$. cenocepacie genes, BCAL2407, a glycosyltransferase presents homology to BB3394.

In order to confirm the role of this cluster of genes in LPS synthesis, we constructed mutants in $B$. bronchiseptica $9.73 \mathrm{H}^{+}$with the individual genes BB3394 and BB3398 interrupted by an antibiotic resistance cassette. Wild type LPS presented a full length LPS with defined bands $\mathrm{A}$ and $\mathrm{B}$ and a diffuse low moving band corresponding to lipid A-core-O antigen structure. As expected, $B b \triangle B B 3398$ LPS showed in Fig. $1 \mathrm{~A}$ a fast moving unique band corresponding to a truncated LPS.

On the other hand, $B b \triangle B B 3394$ LPS profile was indistinguishable from wild type LPS suggesting that deletion of genes involved in GlcN, GlcA or GalNA transfer to the core would not alter the length of the LPS (Fig. 1A). As a first approach to evaluate possible LPS modifications, sensitivity to naive serum were evaluated and compared to wild type strain. Interestingly, $B b \Delta B B 3394$ and $B b \Delta B B 3398$ did not grow properly in naive serum in contrast to $B b 9.73 \mathrm{H}^{+}$strain. Resistance to both agents was restored when $B b \Delta B B 3394$ mutant was complemented with the corresponding gene. Results are shown in Fig. 1B.

To get deeper into the LPSs structures, LPSs from each mutant strain were further purified. The corresponding oligosaccharides were released by acid hydrolysis and analyzed by mass spectrometry. The MALDI-MS spectrum of the $B$. bronchiseptica wild type $9.73 \mathrm{H}^{+}$oligosaccharide in the reflectron positive ion mode using GA as matrix is presented in Fig. 2A and supplemental Fig. S1. In the high molecular weight range a signal at $\mathrm{m} / \mathrm{z} 1637.0$ (calc. $\mathrm{m} / \mathrm{z}$ 1637.5537, $\mathrm{C}_{59} \mathrm{H}_{100} \mathrm{~N}_{5} \mathrm{O}_{46} \mathrm{Na}$ ) consistent with a core fragment containing (Hep)GlcN-(GalNA)Glc-(GlcAGlcNHep)Hep bearing a FucN

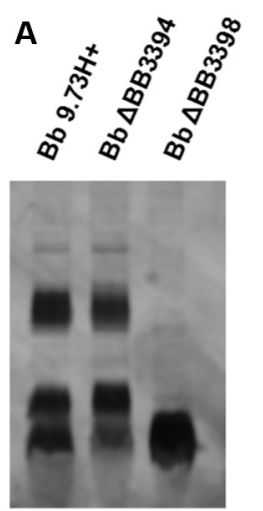

B

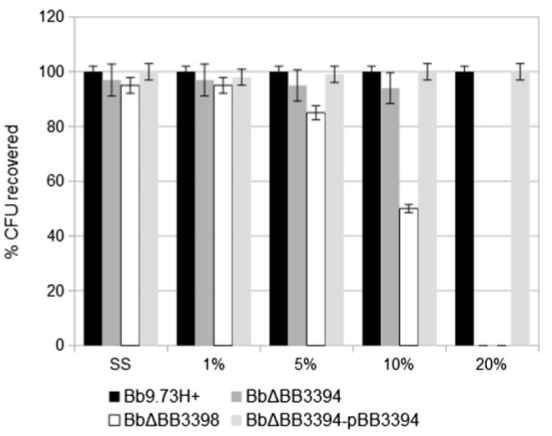

Fig. 1. LPS electrophoretic phenotype reveals modifications in $B b \triangle B B 3398$ but not in $B b \Delta$ BB3394 mutant (A). Silver-stained SDS-PAGE $17.5 \% \mathrm{wt} / \mathrm{vol}$ profiles of phenolwater-extracted LPS samples from Bb9.73 $\mathrm{H}^{+}$wild-type, $B b \Delta B B 3394$ mutant and $B b \Delta B B 3398$ mutant strains. Both mutants showed different susceptibility to naïve sera (B). $\sim 1000 \mathrm{CFU}$ were exposed to naive sera at different proportions to SS media. Appropriate dilutions were plate in BG after $2 \mathrm{~h}$ at $37^{\circ} \mathrm{C}$. Results are expressed as percentage of CFU recovered when were exposed to SS media. * indicates $\mathrm{P}<0.05$; $\mathrm{Bb} 9.73 \mathrm{H}^{+}$versus mutant.
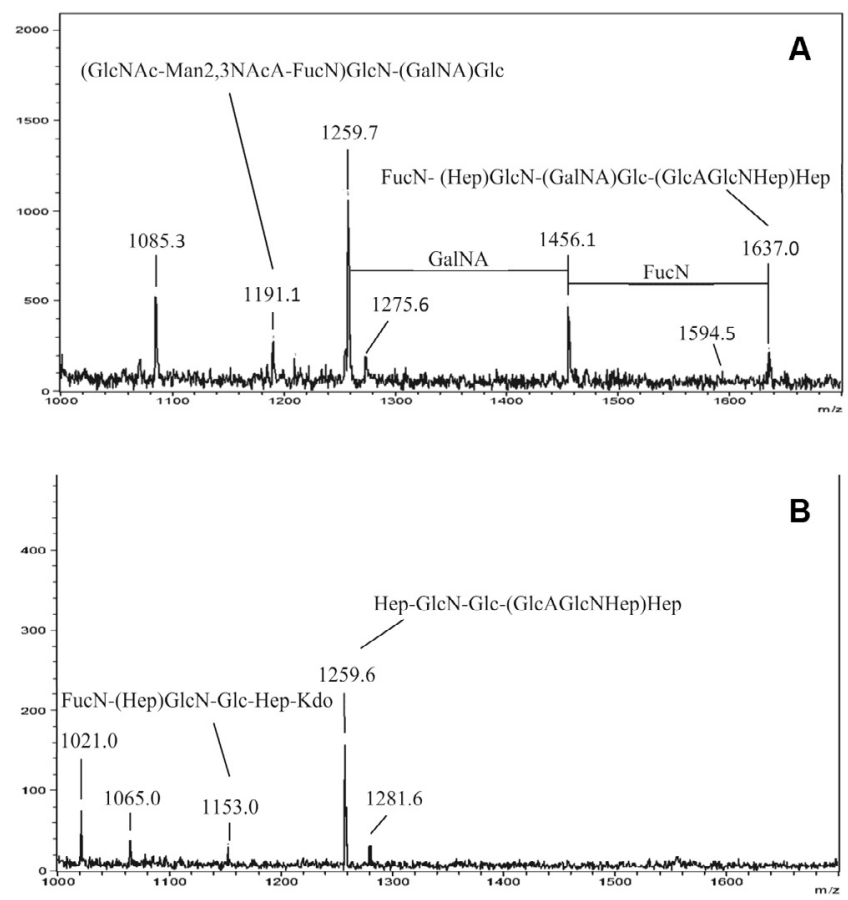

Fig. 2. MALDI-MS spectrum of the B. bronchiseptica wild type $9.73 \mathrm{H}^{+}(\mathrm{A})$ and $B b \triangle B B 3394$ mutant (B) oligosaccharide in the reflectron positive ion mode using GA as matrix

unit from the distal trisaccharide is present. Ion at $m / z 1594.5$ (calc. $\mathrm{m} / \mathrm{z} 1594.5353$ ) would correspond to the loss of an acetyl group from the latter. In accordance, ion at $m / z 1456.1$ (calc $m / z 1456$. $4319, \mathrm{C}_{51} \mathrm{H}_{84} \mathrm{~N}_{3} \mathrm{Na}_{2} \mathrm{O}_{42}$ ) would correspond to the core octasaccharide fragment consistent with (Hep)GlcN-(GalNA)Glc-(GlcAGlcNHep)Hep as an oxonium ion. In addition loss of the GalNA unit from the latter gives rise to $\mathrm{m} / \mathrm{z} 1259.7$ (calc. $\mathrm{m} / \mathrm{z} 1259.4019$, $\mathrm{C}_{45} \mathrm{H}_{76} \mathrm{~N}_{2} \mathrm{NaO}_{37}$ ), and signal at $m / z 1085.3$ (calc. $m / z$ 1084.3656, $\mathrm{C}_{38} \mathrm{H}_{67} \mathrm{~N}_{3} \mathrm{NaO}_{31}$ ) corresponds to the loss of GlcA and a Heptose unit from $\mathrm{m} / \mathrm{z}$ 1456.1. In addition, species at $\mathrm{m} / \mathrm{z} 1191.1$ (calc. $\mathrm{m} / \mathrm{z}$ $1190.4059, \mathrm{C}_{44} \mathrm{H}_{70} \mathrm{~N}_{7} \mathrm{Na}_{2} \mathrm{O}_{29}$ ) is consistent with the distal trisaccha- 
ride (GlcNAc-Man2,3NAcA-FucN) linked to the core fragment GlcN-(GalNA)Glc.

The MALDI-MS spectrum of the mutant BB3394 strain oligosaccharide in the positive ion mode (Fig. 2B and supplemental Fig. S2) showed the highest mass species at $\mathrm{m} / \mathrm{z} 1259.6$ (calc. $\mathrm{m} / \mathrm{z}$ 1260.4103, $\left.\mathrm{C}_{45} \mathrm{H}_{77} \mathrm{~N}_{2} \mathrm{NaO}_{37}\right)$ and $m / z 1281.6(\Delta \mathrm{Na})$ consistent with the core fragment (Hep)GlcN-Glc-(GlcNGlcAHep)Hep. The lack of higher $m / z$ species would indicate the absence of the GalNA unit. In addition, ion at $m / z 1153.0$ (calc. $m / z$ 1153.3997, $\mathrm{C}_{42} \mathrm{H}_{72} \mathrm{~N}_{3}$ $\mathrm{NaO}_{32}$ ) is consistent with a FucN-(Hep)GlcN-Glc-Hep-Kdo core fragment. Ions at $m / z 1065.0$ (calc. $m / z$ 1064.2924, $\mathrm{C}_{35} \mathrm{H}_{61} \mathrm{~N}_{3} \mathrm{Na}_{2}{ }^{-}$ $\left.\mathrm{O}_{29} \mathrm{P}\right)$ and $m / z 1021.0(\Delta 44)$ correspond to FucN-GlcN-Glc-HepKdoP fragment.

The MALDI-MS spectrum of the mutant BB3394 strain, performed in the negative ion mode using nor-harmane as matrix (Fig. 3A and supplemental Fig. S3) showed a complex pattern of signals.

Ion at $m / z 2281.2$ (calc. $m / z 2280.6719, \mathrm{C}_{79} \mathrm{H}_{132} \mathrm{~N}_{7} \mathrm{O}_{65} \mathrm{P}_{2}^{-}$) corresponds to the core region consistent with (Hep)GlcN-Glc(GlcNGlcAHep)Hep-KdoPP linked to the Band A trisaccharide GlcNAc-Man2,3NAcA-FucN. Ion at $\mathrm{m} / \mathrm{z} 1706.9$ (calc. $\mathrm{m} / \mathrm{z}$ 1707.5860, $\left.\mathrm{C}_{64} \mathrm{H}_{103} \mathrm{~N}_{6} \mathrm{O}_{47}\right)$ corresponds to Man2,3NAcAFucNGlcN-Glc(GlcNGlcAHep)Hep-anhKdo. Furthermore, loss of the branching oligosaccharide constituted by (GlcN-GlcA)Hep and a phosphate group from the latter renders ion at $m / z 1130.6$ (calc $m / z$ 1130.4104, $\mathrm{C}_{42} \mathrm{H}_{72} \mathrm{~N}_{3} \mathrm{O}_{32}^{-}$). Signal at $\mathrm{m} / z$ 1840.1 (calc. $\mathrm{m} / \mathrm{z}$ $1839.6312 \mathrm{C}_{68} \mathrm{H}_{112} \mathrm{~N}_{8} \mathrm{O}_{48} \mathrm{P}$ ) would correspond to FucN linked to the Band A trisaccharide plus (Hep)GlcN-Glc-Hep-anhKdoP. Ion at $m / z 1557.1$ (calc. $m / z 1557.6171, \mathrm{C}_{60} \mathrm{H}_{101} \mathrm{~N}_{8} \mathrm{O}_{39}$ ) may be due to prompt fragmentation of $m / z 1840.1$, causing the loss of anhKdoP. In addition, $m / z 1272.2$ (calc. $m / z 1272.3325, \mathrm{C}_{42} \mathrm{H}_{72} \mathrm{~N}_{3} \mathrm{O}_{37} \mathrm{P}_{2}$ ) is consistent with FucN-(Hep)GlcN-Glc-Hep-anhKdoPP. Furthermore, ion at $m / z 1993.7$ (calc. $m / z$ 1994.7606, $\mathrm{C}_{79} \mathrm{H}_{124} \mathrm{~N}_{11} \mathrm{O}_{48}$ ) corresponds to the fragment GlcNAc-Man2,3NAcA-FucN-GlcNAcMan2,3NAcA-FucN-GlcN-Glc-Hep-anhKdo- $\mathrm{H}_{2} \mathrm{O}$; ion at $\mathrm{m} / z 2130.8$
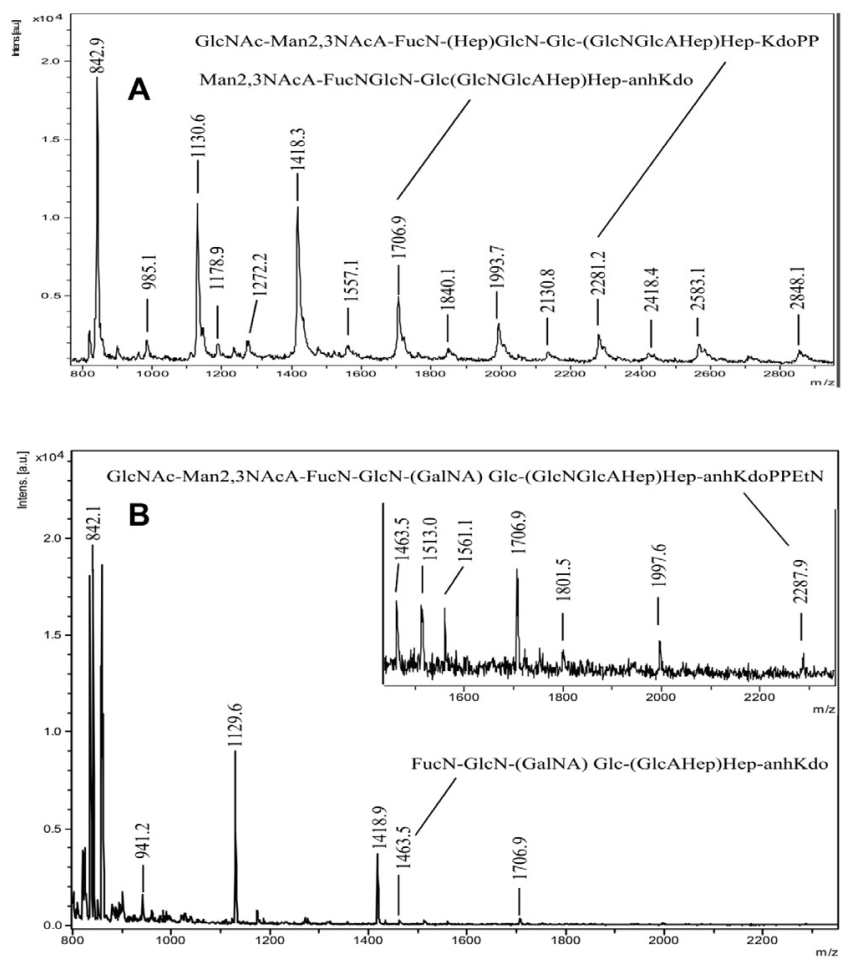

Fig. 3. MALDI-MS spectrum of the mutant $B b \triangle B B 3394$ strain (A) and $B b \Delta B B 3398$ mutant (B) oligosaccharide performed in the negative ion mode. (calc. $m / z$ 2131.6508, $\mathrm{C}_{74} \mathrm{H}_{125} \mathrm{~N}_{8} \mathrm{O}_{59} \mathrm{P}_{2}$ ) would correspond to the trisaccharide unit plus GlcN-Glc-(GlcAGlcNHep)Hep-Kdo-PPEA. In the low MW range, main ion at $m / z 842.9$ (calc. $m / z$ 843.2051, $\mathrm{C}_{28} \mathrm{H}_{46} \mathrm{NO}_{26} \mathrm{P}$ ) may be attributed to the (GlcN-Hep)Hep-anhKdoP fragment. Interestingly no signals corresponding to fragments bearing the GalNA unit could be detected. Conservation of $B B 3394$ in sequenced $B$. bronchiseptica genomes suggests the importance of this substitution for bacteria normal physiology. Resistance to naive sera is a phenotype usually modified when LPS alterations are present. Long LPS structures avoid complement components to reach bacterial surface. However in our hands, a small modification like the absence of the branching GalNA was sufficient to alter resistance to sera.

In several Bordetella LPS, a pentasaccharide fragment that links the 0 -antigen to the core region has been identified. ${ }^{10}$ Previous reports indicated that the 0 -chain of these bacteria LPS was a linear homopolymer of 1,4-linked 2,3-dideoxy-2,3-diacetamido-L-galacturonic acid (L-Gal2,3NAcA). ${ }^{17}$ However, differences between the end groups on $B$. bronchiseptica $\mathrm{O}$ antigens were described such as the presence of a terminal residue (Lac-type, 328.3 Da, and Ala-type, 327.3 Da). Further studies revealed that a proportion of the O-polysaccharide repeating units are present as uronamide (L-Gal2,3NAcAN), the number of which varies between strains. ${ }^{10}$ Also, the presence in the $\mathrm{O}$-antigen of blocks of amides and blocks of acids, whereas less abundant mixed amide/acid ions were evidenced.

In accordance, signals related to the O-antigen oligosaccharide were detected in the MALDI-MS spectrum of the mutant BB3394 strain. Thus, ion peak at $\mathrm{m} / \mathrm{z} 2848.1$ (calc. $\mathrm{m} / \mathrm{z} 2848.0675$, $\mathrm{C}_{111} \mathrm{H}_{163} \mathrm{~N}_{28} \mathrm{O}_{60}$ ) may be attributed to the O-antigen fragment built up of a terminal "Lac type" residue, three uronamide units (L-Gal2,3NAcAN), four 2,3-dideoxy-2,3-diacetamido-L-galacturonic acids (L-Gal2,3NAcA) and three monosaccharides from the linking pentasaccharide (Man2,3NAcAN-Glc2,3NAcAN-GalNAc) with the concomitant loss of water. Ion at $\mathrm{m} / \mathrm{z} 2583.1$ (calc. $\mathrm{m} / \mathrm{z}$ 2582.0044, $\mathrm{C}_{102} \mathrm{H}_{155} \mathrm{~N}_{23} \mathrm{O}_{55}$ ) was assigned to a fragment built up by two 2,3-dideoxy-2,3-diacetamido-L-galacturonic acids (L-Gal2,3NAcA) and one uronamide unit (L-Gal2,3NAcAN) linked to the pentasaccharide plus the Band $A$ trisaccharide unit. Ion at $m / z 2418.4$ (calc. $m / z 2418.9663, \mathrm{C}_{95} \mathrm{H}_{150} \mathrm{~N}_{20} \mathrm{O}_{53}$ ) is consistent with one uronamide unit linked to the pentasaccharide plus Band $\mathrm{A}$ trisaccharide plus Hep-GlcN. In addition, ion at $m / z 1418.3$ (calc. $m / z$ 1418.5691, $\mathrm{C}_{56} \mathrm{H}_{86} \mathrm{~N}_{14} \mathrm{O}_{29}$ ) is attributed to one uronamide unit linked to the pentasaccharide and ion at $m / z 1178.9$ (calc. $m / z$ 1178.4706, $\mathrm{C}_{46} \mathrm{H}_{72} \mathrm{~N}_{11} \mathrm{O}_{25}^{-}$) corresponds to the pentasaccharide unit.

In contrast, regarding mutant BB3398, the MALDI-MS spectrum of the released oligosaccharide, in the negative mode (Fig. 3B and supplemental Fig. S3) showed the highest mass ion at $\mathrm{m} / \mathrm{z} 2287.9$ (calc. $m / z 2288.6883, \mathrm{C}_{80} \mathrm{H}_{132} \mathrm{~N}_{9} \mathrm{O}_{63} \mathrm{P}_{2}$ ) as expected for the lack of the $\mathrm{O}$-antigen structure suggested by SDS-PAGE. This ion corresponds to a GlcNAc-Man2,3NAcA-FucN-GlcN-(GalNA)Glc(GlcNGlcAHep)Hep-anhKdoPPEtN structure. In addition, ion at $m /$ $z 1997.6$ (calc. $\left.m / z 1998.5667 \mathrm{C}_{70} \mathrm{H}_{114} \mathrm{~N}_{7} \mathrm{O}_{58} \mathrm{P}_{2}\right)$ would correspond to Man2,3NAcA-FucN-GlcN-(GalNA)Glc-(GlcN GlcAHep)HepanhKdoPP after the loss of $\mathrm{CO}_{2}(44 \mu)$. Also ion at $m / z 1561.1$ (calc. $m / z$ 1561.4569, $\mathrm{C}_{54} \mathrm{H}_{90} \mathrm{~N}_{4} \mathrm{O}_{46} \mathrm{P}$ ) corresponds to FucN-GlcN-(GalNA) Glc-(GlcAHep)Hep-KdoP. Furthermore, ions at $m / z 1418.9$ (calc. $m /$ $\left.z 1419.4800 \mathrm{C}_{54} \mathrm{H}_{87} \mathrm{~N}_{4} \mathrm{O}_{42}\right)$ and $m / z 1463.5(\Delta 44)$ are ascribed to FucN-GlcN-(GalNA)Glc-(GlcAHep)Hep-anhKdo. In all cases the GalNA unit linked to the Glc of the core region was present in this mutant.

Interestingly, absence of GalNA in BB3394 mutant is not detrimental for distal structures synthesis. However, absence of lateral Heptose in BB3398 LPS generated a rough LPS indicating that Heptose needs to be present to be recognize as a substrate for enzymes 
involved in LPS synthesis. Similar observation was previously reported with core second heptose. ${ }^{11}$ Deletion of waaF an heptosyltransferase involved in second heptose addition generated a deep rough phenotype.

Once confirmed we have $B$. bronchiseptica LPS with deep modifications (deep rough LPS from BbLP39 and $B b \Delta B B 3398$ ) or mildly modifications like $B b \triangle B B 3394$ we hypothesized that structural differences will held different immune responses. We have previously shown that a deep rough LPS triggers different interleukine responses in bone marrow derived dendritic cells. ${ }^{18}$

Dendritic cell response to smooth or complete $B$. bronchiseptica LPS is characterized by high TNF-alpha levels. Same response is observed when wild type LPS is instilled in naïve mice. ${ }^{19}$ Hence we evaluate if LPSs first described in the present work were able to elicit a TNF-alpha response. When LPS derived from BB3398 or BB3394 mutants were intranasally instilled in mice, differences were observed (Table 1 ).

Interestingly, deep rough LPS derived from $B b \Delta \mathrm{BB} 3398$ elicited non significantly differences to wild type LPS. However, we showed that GalNA substitution is important to induce TNF-alpha response. Absence of this lateral sugar in B. bronchiseptica LPS inhibits TNF-alpha response (Table 1). Interestingly, TNF-alpha is an important factor secreted during first steps of infection and its absence is detrimental to limitation of $B$. bronchiseptica infection. ${ }^{19}$

It has been suggested that LPS immune activity may be responsible for directing an appropriated protective response. ${ }^{20,21}$ Therefore, a previously tested vaccination schedule was used to evaluate protection capacity of the different LPSs here in described. ${ }^{22}$ Female three weeks old BALB/c mice were i.p. immunized with $5 \mu \mathrm{g}$ of purified wild type LPS. As a control, whole cell formaldehyde inactivated vaccine was used to immunize mice. After 2 immunizations, mice were challenged intranasally with sublethal doses of wild type $B$. bronchiseptica. Five days after challenge, animals were sacrificed and the number of bacteria in lungs was determinate. As expected not immunized mice presented a high number in CFU/lung, while whole cell immunized mice were protected against infection progress (Fig. 4).

One of main components of whole cell vaccine is LPS. When mice were immunized with $B$. bronchiseptica wild type LPS no significantly differences were observed with mice immunized by whole cell (Fig. 4).

Protection induced by $B$. bronchiseptica LPS was genera and species specific. If we immunized with either B. pertussis or E. coli DH5 $\alpha$ LPS, no protection was observed (Fig. 4). Both E. coli DH5 $\alpha$ and $B$. pertussis LPS are short-chain LPS, commonly named lipooligosaccharide. Interestingly B. pertussis and B. bronchiseptica core structure showed no differences.

When mice were immunized with $5 \mu \mathrm{g}$ of a deep rough LPS from BbLP39 mutant no protection was observed supporting that distal LPS portions are necessary to induce protection (Fig. 4).

Using the same vaccination and challenge schedule, purified LPS from $B b \Delta B B 3398$ and $B b \Delta B B 3394$ with confirmed structure changes were evaluated. As shown in Fig. 4, Bb $\Delta B B 3398$ deep rough LPS was not able to elicit a protective response in mice as the other deep rough LPS from BbLP39. Interestingly, LPS from $B b \triangle B B 3394$ mutant, lacking the GalNA unit in the core, protects mice against infection as the wild type LPS. This result is in agree-

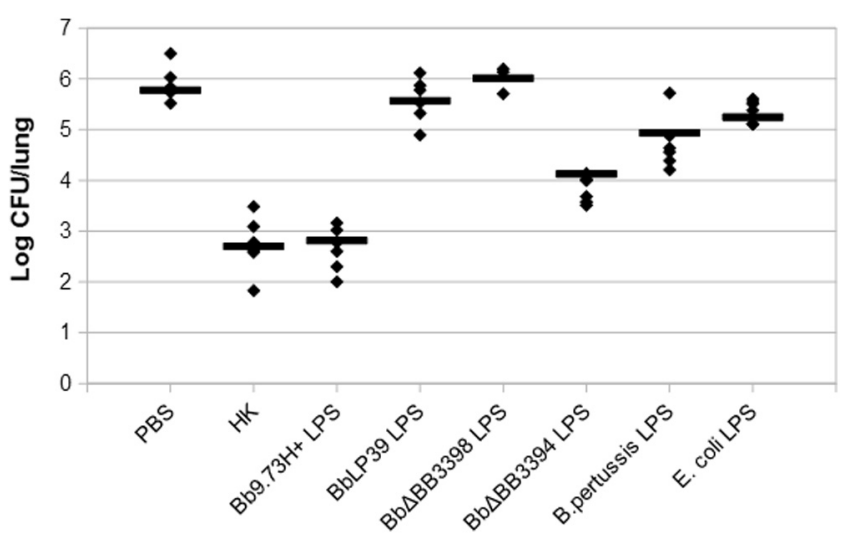

Fig. 4. Effect of systemic (i.p.) immunization with different LPS structures and challenged with wild type $B b 9.73 \mathrm{H}^{+}$in $\mathrm{C} 3 \mathrm{H} / \mathrm{HeN}$ (black columns) or $\mathrm{C} 3 \mathrm{H} / \mathrm{HeJ}$ mice (grey columns). Statistical analysis was performed by ANOVA and Tukey test. * indicate significant differences with $\mathrm{p}<0.001$ respect to $\mathrm{C} 3 \mathrm{H} / \mathrm{HeN}$ mice.

ment to Buboltz and co-workers report. They showed that modifications in distal structures on O-antigen are sufficient to induce different protective responses. ${ }^{23}$ Hence, even somehow core LPS structure can modify immune response, distal portions of LPS like O-antigen are necessary and sufficient to trigger a protective response. These results should be considered to develop a vaccine with cross protection between Bordetella species.

In conclusion, we have identified a new gene involved in the synthesis of the core LPS in B. bronchiseptica. Genes involved in distal LPS structure synthesis have been described previously. However, genes involved in core synthesis remains unknown probably due to dispersal location of genes through the genome. The core is a branched hetero-oligomer. Function of core lateral sugars are unknown. Here we presented first steps in function description, using LPS mutants with different core modifications.

We also demonstrated that LPS modifications correlates with reduction in resistance to naive sera. In addition, differences in immune response to LPS were observed, however lateral modification GalNA was expendable to elicit protection against B. bronchiseptica infection. Further research is needed to establish role of all sugars present in Bordetella LPS. This knowledge will be necessary to design LPS containing vaccines.

\section{Conflict of interest}

The authors declare that they have no conflicts of interest with the contents of this article.

\section{Funding}

This work was supported by University of Buenos Aires, CONICET and the Agencia Nacional de Promoción Científica y Tecnológica (ANCPyT). The Ultraflex II (Bruker) TOF/TOF mass spectrometer was supported by a grant from ANPCYT, PME 125. Authors are member of the Scientific Career of CONICET.

Table 1

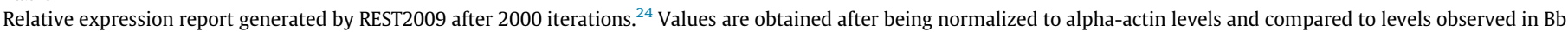
$9.73 \mathrm{H}^{+}$LPS stimulated mice. $\mathrm{P}(\mathrm{H} 1)$ : Probability of alternate hypothesis that difference between sample and control groups is due only to chance.

\begin{tabular}{|c|c|c|c|c|}
\hline & TNF-alpha expression & Std. Error & $\mathrm{P}(\mathrm{H} 1)$ & Result vs $\mathrm{Bb} 9.73 \mathrm{H}^{+}$ \\
\hline LPS $B b \Delta \mathrm{BB} 3398$ & 0.556 & $0.271-1.180$ & 0.138 & \\
\hline LPS $B b \triangle B B 3394$ & 0.242 & $0.119-0.527$ & 0.001 & Down \\
\hline
\end{tabular}




\section{Acknowledgments}

This work was supported by National Agency for the Promotion of Science and Technology (ANCPyT) grants to D.F.H and AC. A.C., D. F.H., J.F. and F.S. are members of the Scientific Career of CONICET.

\section{A. Supplementary material}

Supplementary data associated with this article can be found, in the online version, at http://dx.doi.org/10.1016/j.bmcl.2016.12. 049.

\section{References}

1. Preston A, Mandrell RE, Gibson BW, Apicella MA. Crit Rev Microbiol. 1996;22:139-180.

2. Rietschel ET, Kirikae T, Schade FU, et al. FASEB J. 1994;8:217-225.

3. Trent MS, Stead CM, Tran AX, Hankins JV. J Endotoxin Res. 2006;12:205-223.

4. Harvey HA, Swords WE, Apicella MA. J Autoimmun. 2001;16:257-262.

5. Maskell DJ, Szabo MJ, Butler PD, Williams AE, Moxon ER. Res Microbiol. $1991 ; 142: 719-724$.

6. de Vries FP, van Der Ende A, van Putten JP, Dankert J. Infect Immun. 1996;64:2998-3006.
7. Chmiela M, Miszczyk E, Rudnicka K. World J Gastroenterol. 2014;20:9882-9897.

8. Mann PB, Elder KD, Kennett MJ, Harvill ET. Infect Immun. 2004;72:6650-6658.

9. Le Blay K, Gueirard P, Guiso N, Chaby R. Microbiology. 1997;143:1433-1441.

10. Preston A, Petersen BO, Duus J, et al. J Biol Chem. 2006;281:18135-18144.

11. Allen AG, Isobe T, Maskell DJ. J Bacteriol. 1998;180:35-40.

12. Sisti F, Fernández J, Rodríguez ME, Lagares A, Guiso N, Hozbor DF. Infect Immun. 2002;70:1791-1798.

13. Geurtsen J, Dzieciatkowska $\mathrm{M}$, Steeghs $\mathrm{L}$, et al. Infect Immun. 2009;77:2602-2611.

14. Medini D, Donati C, Tettelin H, Masignani V, Rappuoli R. Curr Opin Genet Dev. 2005; 15:589-594.

15. Hester SE, Park J, Goodfield LL, Feaga HA, Preston A, Harvill ET. BMC Evol Biol. 2013;13:209.

16. Ortega X, Silipo A, Saldías MS, Bates CC, Molinaro A, Valvano MA.J Biol Chem. 2009;284:21738-21751.

17. Vinogradov E, Peppler MS, Perry MB. Eur J Biochem. 2000;267:7230-7237.

18. Sisti F, Fernández J, Higgins SC, et al. Microbiol Immunol. 2011;55:847-854.

19. Mann PB, Wolfe D, Latz E, Golenbock D, Preston A, Harvill ET. Infect Immun 2005;73:8144-8152.

20. Zhang X, Goebel EM, Rodríguez ME, Preston A, Harvill ET. Infect Immun. 2009;77:5050-5058.

21. Errea A, Moreno G, Sisti F, Fernández J, Rumbo M, Hozbor DF. Med Microbio Immunol. 2010;199:103-108.

22. Roberts R, Moreno G, Bottero D, et al. Vaccine. 2008;26:4639-4646.

23. Wolfe DN, Buboltz AM, Harvill ET. PLoS One. 2009;4:e4280.

24. Pfaffl MW. Nucl Acids Res. 2001;29:e45. 